\title{
Multichannel Online Quality and Efficiency Power Network Diagnostic according to IEC Standards
}

\author{
Richárd Bátorfi \\ PHD student \\ Department of Electrical and Electronic Engineering \\ University of Miskolc, Hungary \\ H-3515 Miskolc-Egyetemváros, \\ Phone: +36-46-565-111 / 1144, Fax:+36-46-563-447, e-mail: elkbr@uni-miskolc.hu
}

\begin{abstract}
Today and in the future electrical energy is a significant energy source in all area of life therefore its quality and consumption has more and more importance in practical and also scientific means. The small- and medium-sized enterprises very often do not take care about its quality as they do not understand and realise that their electricity bill also depends on the quality of the energy. IEC Standards describe how to measure the quality of electrical energy and determines the acceptable levels of quality parameters. Using today's advanced computers a more useful measurement system can be created according to IEC standards than other acquirable analysing devices.
\end{abstract}

\section{Key words}

Energy saving, power network, power quality, diagnostic, measuring system

\section{Introduction}

The aim of the research project supported by the Regional University Knowledge Centre of Mechatronics and Logistics Systems in University of Miskolc was development of a highly reliable system suitable for diagnostics of electrical power network of small and medium enterprises (SMEs) according to IEC 61000 standards in order to determine efficiency of the energy utilization of the company. Saving the energy resources, minimizing the energy utilization have one of the highest priority in the European Union development directions in the next years, including the EU7 projects focusing also to this area.

The small- and medium-sized enterprises wrestle for cost-reduction, but very often they do not take care about wasting the electrical energy. One of the regional aims of the Centre is to provide effective support for SMEs of the region in $\mathrm{R} \& \mathrm{D}$ and knowledge-intensive services. One is the most successful service is the diagnostic of the power electrical network of the enterprises in order to define the quality of the energy and also to reduce their energy cost.

\section{Electrical Energy}

Electrical energy is a significant energy source for industrial and commercial applications. This energy looks like a usual good from one side, as it is produced in a plant and transported to the consumers; but it is a very special good from the other side as it is produced at power stations and transported via wiring before it is purchased and used by the consumers. As all the goods in general its quality and quantity can be determined. As the quantity is more visible than the quality and it has direct effect to the cost, in most cases the consumers don't care about the quality of the electrical energy, nevertheless they could reduce the electricity bill if electrical energy was better quality and beyond that the poor quality also can cause damages for equipments. Providing standard quality of electrical energy on the place of utilization is not an easy task, because non-standard energy can not be removed from the network and consumers can also disturb each other operation. The power supplier would like to know how contaminate its power network but the consumers also want to know if they receive the appropriate quality. Hence it is very significant to examine quality of electrical energy and to study the efficiency of energy consumption of company.

Quality problems have particular importance in Hungary, where $10 \%$ of the overall energy consumption is the electrical network loss including those losses resulted from the improper quality of the electrical energy. Table I. shows the overall energy consumption and the electrical network losses in Hungary from 1998 till 2006.

The North-East Hungary has a specially contaminated electrical energy because the significant "old-fashioned" metallurgical industry in the region. The quality problems 
have particular importance in this area especially for the electronics industry, where the technology requires disturbance-free energy source. We use our diagnostic system on this region of Hungary to determine parameters of electrical energy.

Table I. Overall energy consumption and electrical network loss in Hungary

\begin{tabular}{|c|c|c|}
\hline & $\begin{array}{c}\text { Overall energy } \\
\text { consumption } \\
{[\mathrm{GWh}]}\end{array}$ & $\begin{array}{c}\text { Electrical network loss } \\
{[\mathrm{GWh}]}\end{array}$ \\
\hline 1998 & 34998 & 4916 \\
\hline 1999 & 35293 & 4869 \\
\hline 2000 & 35884 & 4733 \\
\hline 2001 & 36872 & 4676 \\
\hline 2002 & 37730 & 4399 \\
\hline 2003 & 38317 & 4240 \\
\hline 2004 & 38453 & 3980 \\
\hline 2005 & 39149 & 3941 \\
\hline 2006 & 40271 & 3964 \\
\hline
\end{tabular}

\section{Effect of Information technology on Research of Electrical Energy}

Nowadays the information technology is developing very rapidly. It has a great impact on other sciences and its development supports growth of all filed of our life. Using computers during measuring and research of electrical energy has increased greatly the efficiency on these fields. Since processor speed and storage size increased considerably the electrical energy can be analysed by a new way.

In 80's analogue devices were used with registers for measuring power quality. Today digital equipments are preferred because of the advantages of digital technique. Standard EN 61000-4-30 and 61000-4-7 define the structure of a full-digital measurement system and set down the measurement methods. These specify more than $18 \mathrm{kHz}$ sample rate on each channel which requires powerful hardware background. Next edition is being published soon which probably will be stricter than the current. Sample rate should be increased in the second edition hence frequencies of typical in nowadays technologies frequencies can not be examined using only $18 \mathrm{kHz}$ sample rate.

Standards and accurate measurements require more and more stronger hardware background which can be realized with only exploiting development of information technology. Although there are a lot of devices operating according to above-mentioned standards we decided to use a personal computer with a data acquisition board. There are a lot of advantages of the PC based system as opposed to other measuring devices. A PC is always more powerful and flexible, and it can be supplemented with other peripherals according to required functions (e.g. WIFI card to transfer results). Its disadvantage is the size but in the most of the cases it's not a concern. The standards don't demand to store every measured data, but only the calculated averages of RMSs, THDs or other values. However we aim to store every measured data because we want to examine the electrical energy in very details. In this case more hundreds storage drives are necessary which are available just now thanks to development of information technology. Processor speeds are also increased greatly so a lot of calculation can execute faster and more effectively than some years ago.

\section{The measurement and analysing system}

Measurement system was developed using hardware and software products of National Instruments (NI). The long term measuring (weeks in IEC 61000-4-30) provides reliable energy cost analysis of the companies, and opening up the possible methods of energy cost reduction. The new application is suitable also for quality analysis of supplied to the company energy according to the standards.

Each measuring system consists of a PC, a measuring card, sensors and transducers. Each measuring computer uses NI's data acquisition board with at least 15 analogue inputs, so 15 channels: 3 voltages and 4 three phase currents, all over 12 currents can be examined by one PC. Of course number of channels can be easily increased. Outputs of different sensors are connected via converters and via analogue filters to analogue inputs of the card. The applied card determines the number of analogue inputs, the quality of digitalisation including $\mathrm{A} / \mathrm{D}$ resolution and the maximum sampling rate. If one of these parameters is not enough for a specific test we can swap the card for another one. There is not necessary to obtain a new PC but we have to make some modifications in the control software.

Our well-designed and implemented application has two different tasks: measuring (control of the measurement process and data storage) and analysis (data processing). Data acquisition can be performed according to standards or a different from the standard measuring process also can be set if necessary. Sometimes special situations can demand stricter or different measurements. Data processing can run during measuring or at a later date after the measurement. As there is not enough time to calculate all power parameters on-line, measured values are stored on hard drive. On-line calculated parameters depend on our computer's performance. For example if the measurement is running on high speed, we can watch RMS values but nothing else hence there is no more CPU time to calculate other parameters. Since all data is on storage all parameters can be determined at a later time and each measured value and sine period can be examined. Storing all data is not a problem anymore as more hundred gigabytes storage easily available today.

The application calculates and displays the following values and changes of these values in time according to IEC 61000-4-30 and IEC 61000-4-7:

- Power frequency

- RMS values for all channels (voltages, currents);

- Supply voltage dips and swells 
- Voltage interruptions

- Supply voltage unbalance

- Amplitude - frequency spectrum (voltage harmonics and interharmonics) and THD values for all channels;

- $\cos (\varphi)$ values for channel-pairs (U -I);

- Apparent, active and reactive power for channelpairs (U -I);

- Statistical distribution of any properties for any time interval

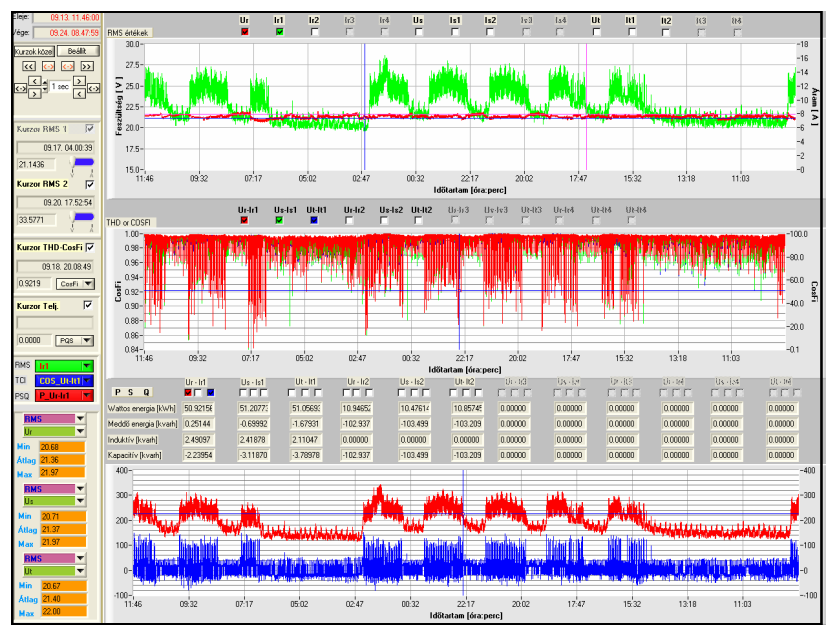

Figure 1. Display of Diagnostic System

Figure 1. shows the main screen of our own developed program. It's easy to use for anybody. Colourful graphs help to separate the different signals and cursors simplify reading values. Another advantage of $\mathrm{PC}$ is that any kind of display can be connected to it therefore we could view the results on a huge screen. Top chart shows RMS, middle one shows THDs or $\cos (\varphi)$ and the bottom shows different power values all in the same time interval. According to EN 61000-4-30 values have to be calculated for 3 seconds, 10 minutes and 2 hours. Our application can compute values not only for these three intervals but for another 14 from one period to one day. Some short time $(0.1-0.2 \mathrm{sec})$ disturbances can not be determined even in 3 second averaging RMSs data, nevertheless they cause significant problems in industrial process control equipment. See Figure 2.

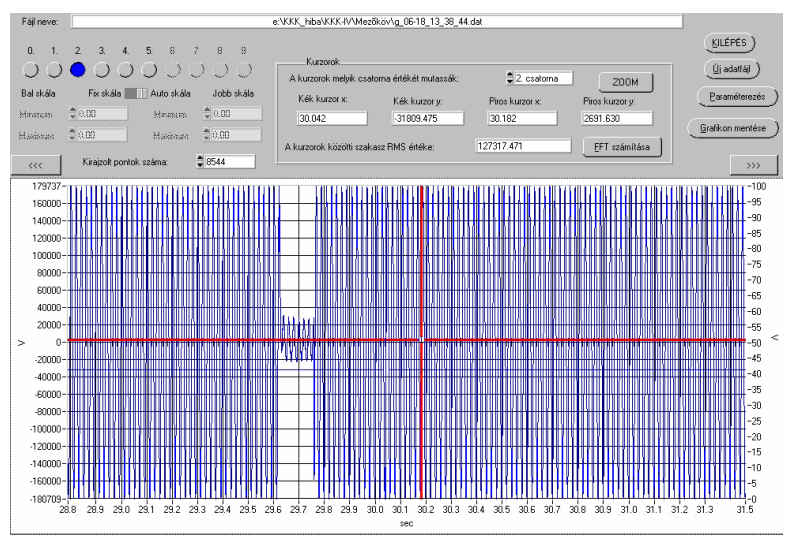

Figure 2. Short time voltage drop measured in a plant
Maximum, minimum and average values also can be read from the display. Statistic graphs are displayed on Figure 3. Statistical distribution can be created for any values for any time interval. It's easy to compare to distribution of RMS voltage values on the three graphs three-phase system.

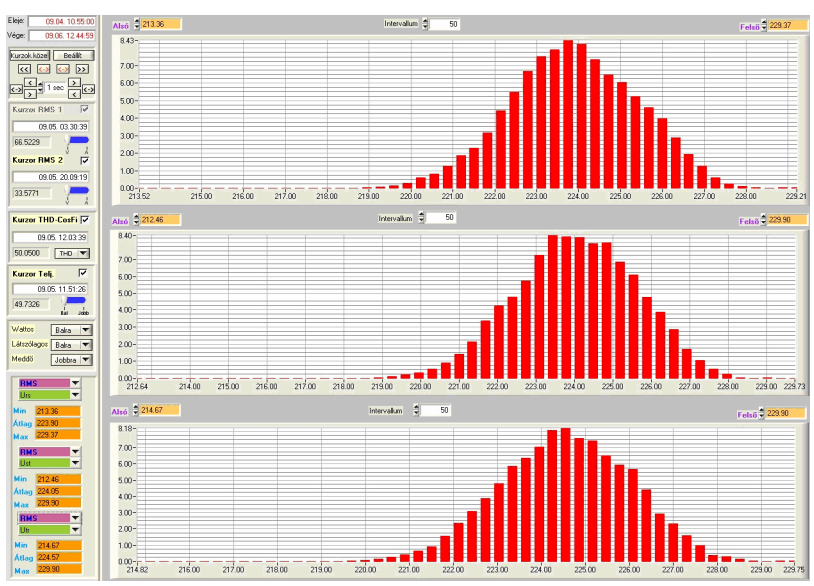

Figure 3. Statistic graphs

Time signals can be visualized at any time at any channel since all data is on the computer's hard drive therefore our measurement system is preferable to other concurrent products. Any distortion can be noticed easily, for example if there is a dip on RMS or THD value is higher than the limit probably voltage time signal has distortion. On Figure 4. the first graph shows voltage waveforms the second graph shows the amplitude-frequency spectrum of either voltage waveform.

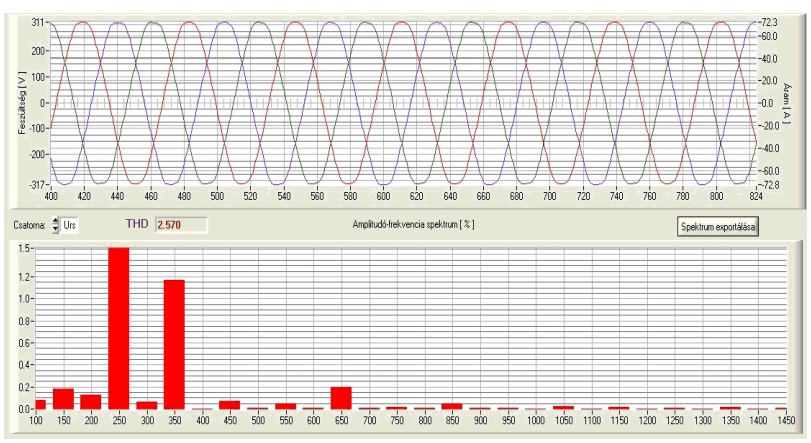

Figure 4. Voltage time signals and amplitude-frequency graph

Figure 5. demonstrates very deformed voltage waves. It was measured in a real industrial environment. It was the worst quality we have ever found in North-East Hungary.

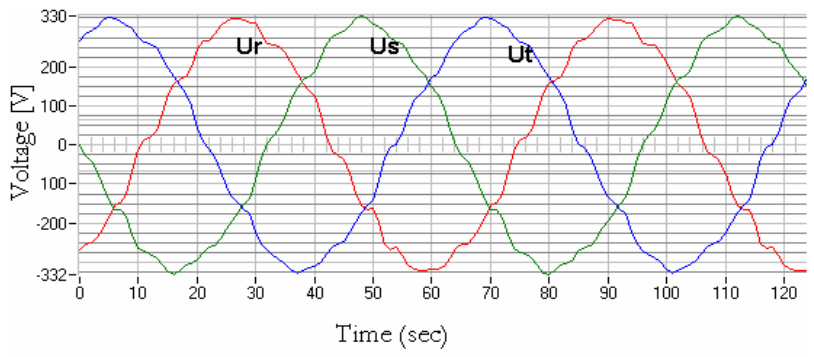

Figure 5. Voltage time signals, disturbances on the electrical network 


\section{Results of the diagnostics}

The release system was tested on electrical power network of nearly 20 companies and one public institution, also suggestions for network changes were provided to them in order to reduce energy costs. Some results are shown on Figure 6. and 7. Experiences of the real tests stimulated performing some modifications on the system, particularly in analyzing software. Now wide range of industrials and institutional networks can be studied by complete system and we are continuing measuring on electrical power network of different companies.

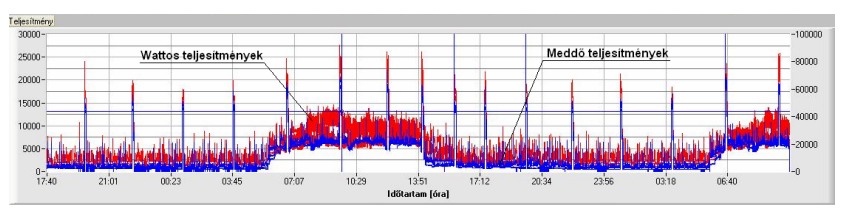

Figure 6. Active (red diagram - left axis) and reactive (blue diagram - right axis) powers in an office building

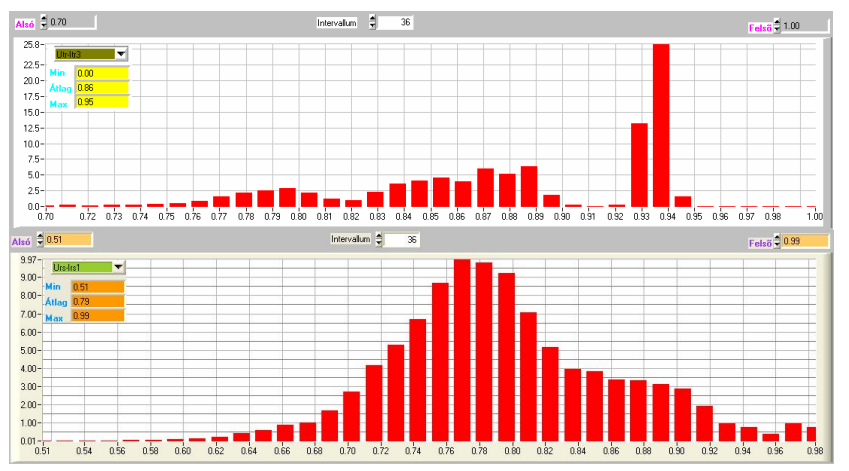

Figure 7. Statistics of $\cos \varphi$ in a good and a poor compensated industrial plant

\section{Future development}

We continue the research and development of the whole system and we have several ideas for increasing its usefulness and efficiency:

$>$ Remote control, remote data processing.

$>$ Increasing speed of evaluation using artificial intelligent methods.

It is expedient if the measurement system can be reach via internet. In this stage we have no any information on the operation of our test system which is usually situated more hundreds kilometres from our institution and runs 10-20-30 days continuously. If any problem occurs during the test we can't do anything and the test must be repeated, wasting time and cost. If our system could send status reports and/or emergency signals, it would highly increase the reliability of the system. Controlling it via internet would also provide possibility of receiving some already available data, characteristic parameters providing remote in-process system diagnostics. WIFI or GPRS technologies are considered since it's not sure that wire network is installed on its district.
The more complex part of the development is the artificial data-mining. As we know from the practice, the evaluation can take very long time. More thousands of data has to be investigated which is a very tiring and long process for a person or a team. Using artificial intelligent methods for evaluation the efficiency of evaluation can be appreciably increased. If the application could find known patterns in time signals the source of the distortion can be discovered easily.

\section{Summary}

Mixing the Information Technology with Power Quality Analysing a highly advanced expert system can be created. In the future the electrical energy will still be one of the most important power source therefore its quality analysis are more important than ever. We reached quite good results with the current system in different companies. Although our system measures complying IEC standards it also can use different or stricter measure methods. Case of changing standard our system could follow the standard changes by modification of program or replacing some parts of the hardware.

\section{Acknowledgement}

The research team of the Department of Electrical and Electronics Engineering of the University of Miskolc expresses the sincerely thanks to National Instruments providing free software package and also to the Mechatronics and Logistics Regional Knowledge Centre, to Pázmány Péter Programme and to KPI and NKTH for the support of this research work.

\section{References}

[1] Kovács E., Szarka T., Radács L., Váradiné Sz. A.: Measurement Methods for Quality Assurance of Electrical Energy Supply int he NorthHungariannRegion. International Conference on Electrical Drives and Power Electronics, Slovakia, 2003. pp.428-432.

[2] Váradiné Sz.A., Szarka T., Szentirmai L.: Electrical Energy Quality and Drive Operation. EPQ 2005. International Scientific and Technical Conference, Efficiency and Power Quality of Electrical Supply of Industrial Enterprises, Mariupol, Ukraine 2005. pp.26-29.

[3] IEC 61000-4-30:2003 Electromagnetic compatibility (EMC) Part 4-30: Testing and measurement techniques - Power quality measurement methods

[4] IEC 61000-4-7:2003 Electromagnetic compatibility (EMC) Part 4-7

[5] Unhauzer Attila: Development of Digital Flicker Analyser for Electrical Network Diagnostic System of Special Requirements, Nagyvárad, 2007, XVII. SzámOkt - VIII. ENELKO, pp 194197 